\title{
GTRD - an integrated view on transcription regulation
}

Fedor A. Kolpakov

Institute of Computational

Technologies SB RAS

BIOSOFT.RU, LLC

Novosibirsk, Russia

fkolpakov@gmail.com

Ivan S. Evshin

Institute of Computational

Technologies SB RAS

BIOSOFT.RU, LLC

Novosibirsk, Russia

ivan@biosoft.ru
Semyon K. Kolmykov

FRC Institute of Cytology and Genetics SB RAS

Institute of Computational

Technologies SB RAS

Novosibirsk, Russia

kolmykovsk@gmail.com

Yury V. Kondrakhin

Institute of Computational

Technologies SB RAS

Novosibirsk, Russia

yvkondrat@mail.ru
Mikhail A. Kulyashov

Novosibirsk State University

Institute of Computational

Technologies SB RAS

Novosibirsk, Russia

m.kulyashov@mail.ru

Ruslan N.Sharipov

Novosibirsk State University Institute of Computational BIOSOFT.RU, LLC

Novosibirsk, Russia

shrus79@biosoft.ru

\begin{abstract}
GTRD - Gene Transcription Regulation Database (http://gtrd.biouml.org/) contains uniformly annotated and processed NGS data (ChIP-seq, ChIP-exo, DNase-seq, MNase-seq, ATAC-seq and RNA-seq) related with transcription regulation. All cell types (cell lines and tissues) presented in the GTRD were arranged into a dictionary, linked with specialised ontologies (BRENDA, Cell ontology, Uberon, Celosaurus, Experimental factor ontology) and further linked with experiments in specialised databases on transcription regulation: FANTOM 5, ENCODE and GTEx. For integrated view in transcription regulation we have developed webinterface that provides browsing, advanced search possibilities, an integrated genome browser and integrated reports by given cell type, transcription factor or gene.
\end{abstract}

Keywords - GTRD, transcription regulation, NGS, uniformed analysis, data integration

\section{Introduction}

Transcription regulation is a complex process that involves a lot of participants. There is a lot of experimental data of different types obtained for different cell types and different experimental conditions which are stored in different databases.

An important task is integration of such data for a given cell type and experimental condition to get an integrated view on main events related with transcription regulation. This is one of the main goals of GTRD database [1].

\section{Results}

The suggested approach is shown on figure 1:

1) we are collecting and uniformly process[1] following NGS data:

- ChIP, Chip-exo - to identify binding regions for transcription factors (TFs) and their coactivators, as well as to identify regions with corresponding histone marks.
- DNase-seq, MNase-seq and ATAC-seq - to identify genome regions with open chromatin and footprints for TF binding sites;

- RNA-seq data where gene expression was changed by TFs activation or knockout to identify genes regulated by these TFs.

2) all cell types (cell lines and tissues) presented in the GTRD were arranged into a dictionary, linked with specialised ontologies (BRENDA, Cell ontology, Uberon, Celosaurus, Experimental factor ontology) and further linked with experiments in specialised databases on transcription regulation: FANTOM 5, ENCODE and GTEx [2].

3) for integrated view in transcription regulation we have developed web-interface:

- based on BioUML platform[3] - it provides browsing and displaying information, advanced search possibilities and an integrated genome browser;

- based on BeanExplorer technology[4] - it provides integrated reports by given cell type, TF or gene.

\section{ACKNOWLEDGMENT}

This study was supported by the Russian Science Foundation, grant No. 19-14-00295.

\section{REFERENCES}

[1] Yevshin I, Sharipov R, Kolmykov S, Kondrakhin Y, Kolpakov F. GTRD: a database on gene transcription regulation - 2019 update. Nucleic acids research. 2019 Jan 8;47(D1):D100-5.

[2] Kulyashov M., Kolmykov S., Yevshin I., Kolpakov F. "Description, Characteristic And Algorithm For Creation Of A Dictionary Of Cell Types And Tissues In The Gtrd Database", CEUR-Workshop proceedings, 2020, Vol-2569, p.13-18.

[3] Kolpakov F, Akberdin I, Kashapov T, Kiselev L, Kolmykov S, Kondrakhin Y, Kutumova E, Mandrik N, Pintus S, Ryabova A, Sharipov R. BioUML: an integrated environment for systems biology and collaborative analysis of biomedical data. Nucleic acids research. 2019 Jul 2;47(W1):W225-33.

[4] Bean Explorer https://github.com/DevelopmentOnTheEdge/be5 


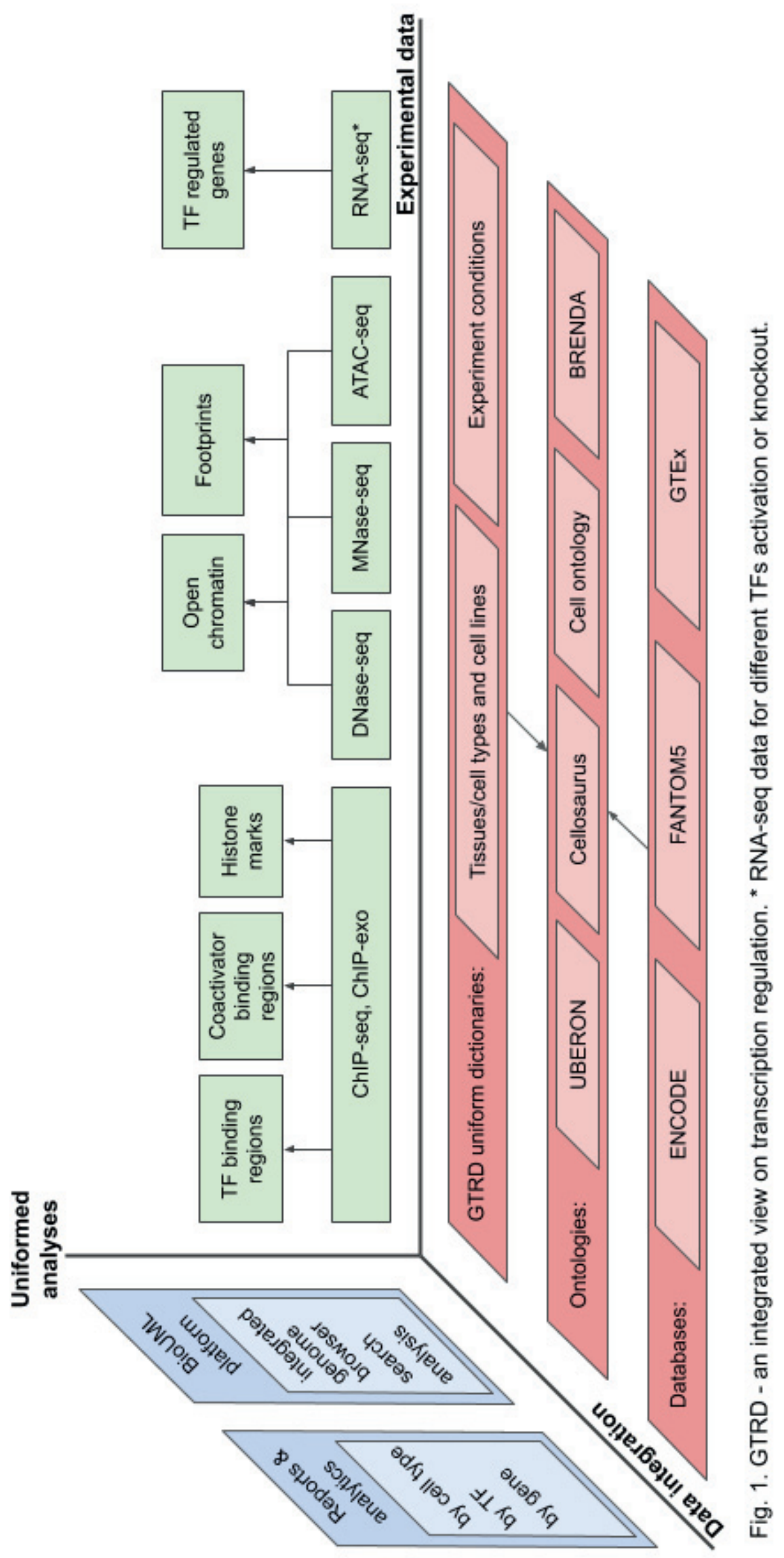

